\title{
Hymenobacter rigui sp. nov., isolated from wetland freshwater
}

Correspondence Jongsik Chun jchun@snu.ac.kr

\author{
Keun Sik Baik, ${ }^{1}$ Chi Nam Seong, ${ }^{1}$ Eun Young Moon, ${ }^{2}$ Yoon-Dong Park, ${ }^{2}$ \\ Hana $\mathrm{Yi}^{2}$ and Jongsik Chun ${ }^{2}$
${ }^{1}$ Department of Biology, College of Natural Sciences, Sunchon National University, Sunchon 540-742, Republic of Korea
${ }^{2}$ School of Biological Sciences and Institute of Microbiology, Seoul National University, NS70, 56-1 Shillim-dong, Kwanak-gu, Seoul 151-742, Republic of Korea

The genus Hymenobacter Hirsch et al. 1998 represents a deep branch within the phylum Bacteroidetes and displays a distinct taxonomic status in view of the high $\mathrm{G}+\mathrm{C}$ content of genomic DNA (55-65 mol\%) of Hymenobacter species, which is nearly unique in this phylum. Currently, there are three species in the genus, namely Hymenobacter roseosalivarius (Hirsch et al., 1998), Hymenobacter actinosclerus (Collins et al., 2000) and Hymenobacter aerophilus (Buczolits et al., 2002). In the course of our study on wetland microbial diversity, a Hymenobacter-like bacterial strain, designated WPCB $131^{\mathrm{T}}$, was isolated from a freshwater sample and was the subject of a taxonomic investigation.

Strain WPCB $131^{\mathrm{T}}$ was isolated from a freshwater sample collected from the wetland in Woopo $\left(35^{\circ} 33^{\prime} \mathrm{N} 128^{\circ} 25^{\prime} \mathrm{E}\right)$, Republic of Korea, during July 2000 using the standard dilution plating technique. Isolation was achieved using PYGV (Staley, 1968) at $25^{\circ} \mathrm{C}$ for 7 days. The isolate was routinely cultured on $\mathrm{R} 2 \mathrm{~A}$ (Oxoid) and maintained as a glycerol suspension $(20 \%, \mathrm{w} / \mathrm{v})$ at $-80^{\circ} \mathrm{C}$.

Bacterial DNA preparation, PCR amplification and sequencing of the 16S rRNA gene were carried out as described previously (Chun \& Goodfellow, 1995). The resultant

The GenBank/EMBL/DDBJ accession number for the 16S rRNA gene sequence of strain WPCB $131^{\top}$ is Da089669. sequence of strain WPCB $131^{\mathrm{T}}$ was aligned manually against sequences obtained from GenBank. Phylogenetic trees were inferred from the regions available for all sequences (positions 43-1438, Escherichia coli numbering system) using the maximum-likelihood (Felsenstein, 1981), maximum-parsimony (Fitch, 1971) and neighbour-joining (Saitou \& Nei, 1987) methods. Evolutionary distance matrices were generated according to Jukes \& Cantor (1969). The resultant neighbour-joining tree topology was evaluated by bootstrap analyses (Felsenstein, 1985) based on 1000 resamplings. Alignment and phylogenetic analyses were carried out using the program jPHYDIT (Jeon et al., 2005) and PAUP 4.0 (Swofford, 1998) as described previously (Yi \& Chun, 2006).

Preliminary sequence comparison with $16 \mathrm{~S}$ rRNA gene sequences held in GenBank indicated that our isolate was closely related to members of the genus Hymenobacter. The newly determined sequence was then aligned manually against representatives of the genus Hymenobacter. Strain WPCB $131^{\mathrm{T}}$ showed the highest $16 \mathrm{~S}$ rRNA gene sequence similarity to $H$. actinosclerus CCUG $39621^{\mathrm{T}}(95 \cdot 5 \%)$; this value is well below the threshold for demarcating bacterial species (Stackebrandt \& Goebel, 1994). Similarities between the $16 \mathrm{~S}$ rRNA gene sequence of strain $\mathrm{WPCB} 131^{\mathrm{T}}$ and those of $H$. aerophilus DSM $13611^{\mathrm{T}}$ and $H$. roseosalivarius DSM $11622^{\mathrm{T}}$ were $94 \cdot 5$ and $93 \cdot 2 \%$, respectively. No other 
described bacterial species showed more than $90 \% 16 \mathrm{~S}$ rRNA gene sequence similarity to our isolate. This relationship between our isolate and other members of the genus Hymenobacter is also evident in the phylogenetic tree. Strain $\mathrm{WPCB} 131^{\mathrm{T}}$ represented a distinct phyletic line within the genus Hymenobacter and formed a robust monophyletic clade together with other hymenobacters in all trees inferred in this study (Fig. 1). It is clear from 16S rRNA gene sequence similarity data and phylogenetic analysis that strain $\mathrm{WPCB} 131^{\mathrm{T}}$ represents a novel species in the genus Hymenobacter.

Growth on various standard bacteriological media was tested using nutrient agar (NA; Difco), tryptone soy agar (TSA; Oxoid) and R2A agar (Oxoid). Cells grown on TSA at $28^{\circ} \mathrm{C}$ for 3-7 days were used for physiological and biochemical tests. Motility was examined by phase-contrast microscopy using wet mounts. Growth in various $\mathrm{pH}$ ( $\mathrm{pH} 4-12)$, temperature $\left(4-42^{\circ} \mathrm{C}\right)$ and $\mathrm{NaCl}$ concentration $(0,1,2,3,5$ and $10 \%, w / v)$ ranges was tested using TSA for up to 1 week. Anaerobic growth was checked in an anaerobic cabinet $\left(\mathrm{CO}_{2} / \mathrm{H}_{2} / \mathrm{N}_{2}, 10: 10: 80\right.$ by vol.; Sheldon Manufacturing) using TSA. Catalase and oxidase activities were determined using 3\%(v/v) hydrogen peroxide and Kovacs' reagent (Kovacs, 1956), respectively. Other physiological and biochemical tests were performed using API 20E, API 20NE and API 50CHB (bioMérieux) test panels. Enzyme activities were tested using the API ZYM kit (bioMérieux) following the manufacturer's instructions. The detailed results of physiological and biochemical analyses are given in Table 1 and the species description. It is evident from Table 1 that there are several phenotypic characters that readily differentiate strain $\mathrm{WPCB} 131^{\mathrm{T}}$ from other Hymenobacter species.

Cellular fatty acids of strain WPCB $131^{\mathrm{T}}$ were analysed as methyl esters by GLC according to the instructions of the Microbial Identification System (MIDI). The G + C content of the DNA was determined using the thermal denaturation
Table 1. Phenotypic characteristics that differentiate strain WPCB $131^{\top}$ from other Hymenobacter species

Species/strains: 1, WPCB131 ${ }^{\mathrm{T}}$; 2, H. actinosclerus; 3, H. aerophilus; 4, H. roseosalivarius. Data from Hirsch et al. (1998), Collins et al. (2000), Buczolits et al. (2002) and this study. +, Positive; -, negative; $\mathrm{W}$, weakly positive; ND, not determined.

\begin{tabular}{|c|c|c|c|c|}
\hline Characteristic & 1 & 2 & 3 & 4 \\
\hline Pigmentation & Pinkish-red & Red & Red & Reddish \\
\hline \multicolumn{5}{|l|}{ Growth at $\left({ }^{\circ} \mathrm{C}\right)$ : } \\
\hline 4 & + & - & + & + \\
\hline 28 & + & + & + & - \\
\hline 37 & + & + & - & - \\
\hline 40 & - & + & - & - \\
\hline 42 & - & + & - & - \\
\hline Oxidase (Kovacs' reagent) & - & + & + & + \\
\hline \multicolumn{5}{|l|}{ Assimilation of: } \\
\hline $\mathrm{N}$-Acetyl-D-glucosamine & - & + & - & - \\
\hline Arbutin & - & + & - & - \\
\hline D-Cellobiose & + & + & - & - \\
\hline D-Fructose & + & - & + & - \\
\hline Gluconate & - & + & - & - \\
\hline D-Glucose & + & + & + & - \\
\hline D-Mannose & - & + & + & - \\
\hline D-Maltose & + & - & - & - \\
\hline D-Ribose & - & + & - & - \\
\hline Sucrose & + & - & + & - \\
\hline Salicin & $\mathrm{W}$ & - & - & - \\
\hline D-Trehalose & + & - & - & - \\
\hline \multicolumn{5}{|l|}{ Enzyme activity (API ZYM): } \\
\hline$N$-Acetyl- $\beta$-glucosaminidase & + & $\mathrm{ND}$ & - & $\mathrm{ND}$ \\
\hline Cystine arylamidase & $\mathrm{W}$ & + & - & ND \\
\hline DNA G $+\mathrm{C}$ content $(\mathrm{mol} \%)$ & 65 & 62 & 63 & 56 \\
\hline
\end{tabular}

method of Marmur \& Doty (1962). The cellular fatty acid composition of strain WPCB $131^{\mathrm{T}}$ was similar to those of other Hymenobacter species, but differed from them in

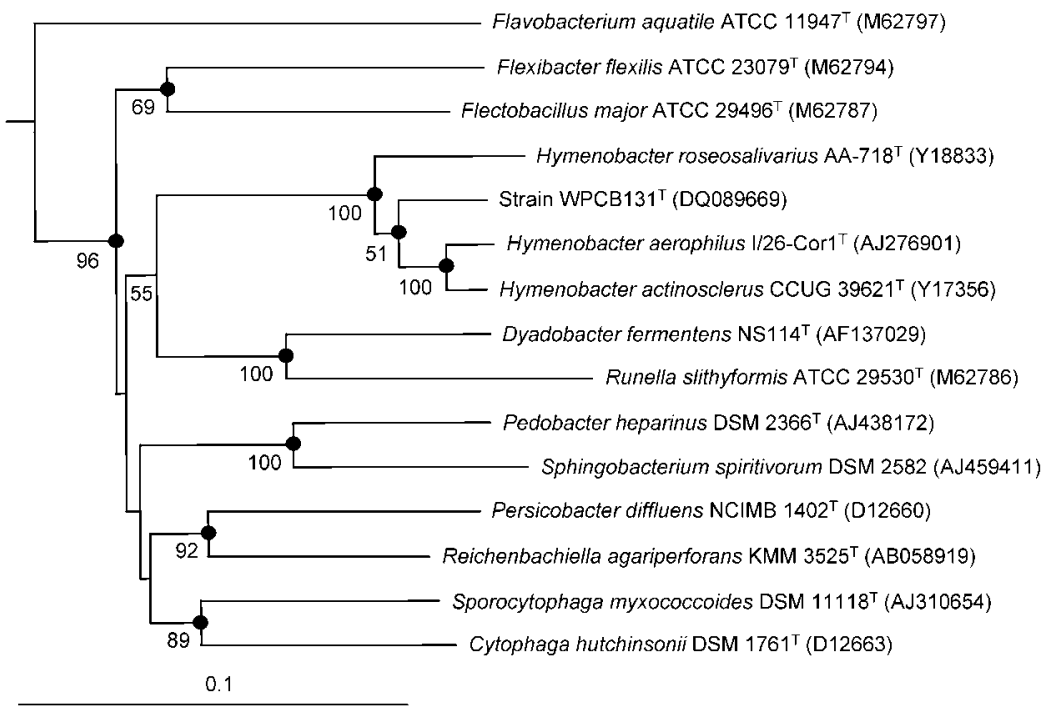

Fig. 1. Neighbour-joining tree based on nearly complete 16S rRNA gene sequences showing relationships between strain $\mathrm{WPCB} 131^{\top}$ and related taxa within the phylum Bacteroidetes. Numbers at the nodes are the percentages of bootstrap support based on neighbour-joining analyses of 1000 resampled datasets; solid circles indicate that the corresponding nodes (groupings) are also recovered in maximumlikelihood and maximum-parsimony trees. Helicobacter pylori ATCC $43504^{\top}$ (U01330) was used as an outgroup. Bar, $0 \cdot 1$ nucleotide substitution per position. 
quantities, especially for unsaturated $\left(\mathrm{C}_{15: 0}\right)$ and monosaturated $\left(\mathrm{C}_{16: 1}\right)$ fatty acids (Table 2$)$. The DNA G+C content of strain $\mathrm{WPCB} 131^{\mathrm{T}}$ was $65 \mathrm{~mol} \%$.

Formation of a distinctive phyletic line within the genus Hymenobacter indicates that strain $\mathrm{WPCB} 131^{\mathrm{T}}$ can be assigned as a representative of a novel species in the genus Hymenobacter. In addition, a number of physiological and chemotaxonomic characters clearly distinguish this isolate from other Hymenobacter species (Tables 1 and 2). Therefore, strain $\mathrm{WPCB} 131^{\mathrm{T}}$ should be classified as a member of a novel species within the genus Hymenobacter, for which the name Hymenobacter rigui sp. nov. is proposed.

\section{Description of Hymenobacter rigui sp. nov.}

Hymenobacter rigui (ri'gu.i. L. gen. n. rigui of a well-watered place).

Cells are rod-shaped, Gram-negative, aerobic, non-sporeforming and produce water-insoluble pinkish-red pigment. Motility is not observed. Cells grow best on R2A and TSA; weak growth is observed on NA. Colonies on TSA are translucent, low-convex, circular, smooth and slimy; diameter is up to $3.0 \mathrm{~mm}$ after 5 days at $25-30{ }^{\circ} \mathrm{C}$ ( $\mathrm{pH} 7)$. Growth occurs in $0-2 \%(\mathrm{w} / \mathrm{v}) \mathrm{NaCl}$ (optimum $0 \%$ ), $\mathrm{pH} 5-11$ (optimum $\mathrm{pH}$ 6) and at $4-37^{\circ} \mathrm{C}$ (optimum $30{ }^{\circ} \mathrm{C}$ ). Oxidase-negative and catalase-positive. Does not reduce nitrate to nitrite. Aesculin hydrolysis is weakly positive. Does not utilize citrate. Negative for fermentation of glucose. Produces gelatinase, but not arginine dihydrolase, urease, lysine decarboxylase, ornithine decarboxylase, tryptophan deaminase, $\mathrm{H}_{2} \mathrm{~S}$, indole or acetoin. Produces alkaline phosphatase, esterase (C4), esterase lipase (C8), leucine arylamidase, acid phosphatase, naphthol-AS-BIphosphohydrolase, valine arylamidase and $\alpha$-glucosidase, but not lipase (C14), trypsin, $\alpha$-chymotrypsin, $\beta$-glucuronidase, $\alpha$-galactosidase, $\beta$-galactosidase, $\beta$-glucosidase, $\alpha$ mannosidase or $\alpha$-fucosidase. Utilizes galactose, aesculin, starch, lactose, inulin, melezitose, raffinose, glycogen and gentiobiose as sole carbon and energy sources. Does not utilize glycerol, erythritol, D-arabinose, L-arabinose, ribose, D-xylose, L-xylose, adonitol, methyl $\beta$-D-xylopyranoside, sorbose, rhamnose, dulcitol, inositol, D-mannitol, Dsorbitol, methyl $\alpha$-D-mannopyranoside, methyl $\alpha$-D-glucopyranoside, $N$-acetylglucosamine, amygdalin, arbutin, melibiose, xylitol, D-turanose, D-lyxose, D-tagatose, Dfucose, L-fucose, D-arabitol, L-arabitol or gluconate. Other physiological and biochemical characteristics are given in Table 1 . Major fatty acids are iso- $\mathrm{C}_{15: 0}(34 \cdot 8 \%), \mathrm{C}_{16: 1} 105 \mathrm{c}$ $(15 \cdot 0 \%)$, anteiso- $\mathrm{C}_{17: 1} \quad \mathrm{~B} /$ iso- $_{17: 1}$ I $(14 \cdot 4 \%)$ and $\mathrm{C}_{16: 1} \omega 7 c /$ iso- $\mathrm{C}_{15: 0} 2-\mathrm{OH}(13 \cdot 8 \%)$; complete fatty acid data are given in Table 2.

The type strain is WPCB131 $1^{\mathrm{T}}\left(=\mathrm{IMSNU} 14116^{\mathrm{T}}=\mathrm{KCTC}\right.$ $12533^{\mathrm{T}}=$ NBRC $101118^{\mathrm{T}}$ ), isolated from freshwater of Woopo wetland, Republic of Korea. The DNA G+C content of strain WPCB $131^{\mathrm{T}}$ is $65 \mathrm{~mol} \%$.
Table 2. Cellular fatty acid content (\%) of strain WPCB $131^{\top}$ and the type strains of Hymenobacter species

Strains: 1, WPCB131 ${ }^{\mathrm{T}}$; 2, H. actinosclerus; 3. H. aerophilus; 4, H. roseosalivarius. Data from Hirsch et al. (1998), Collins et al. (2000), Buczolits et al. (2002) and this study. Values are percentages of total fatty acids. Tr, $<1 \cdot 0 \%$; ND, not detected.

\begin{tabular}{|c|c|c|c|c|}
\hline Characteristic & 1 & 2 & 3 & 4 \\
\hline Summed feature $2^{\star}$ & ND & $2 \cdot 3$ & $\operatorname{Tr}$ & ND \\
\hline iso- $\mathrm{C}_{15: 0}$ & $34 \cdot 8$ & $22 \cdot 3$ & $10 \cdot 8$ & $8 \cdot 3$ \\
\hline anteiso- $C_{15: 0}$ & $5 \cdot 9$ & $25 \cdot 8$ & $22 \cdot 3$ & ND \\
\hline $\mathrm{C}_{15: 1} \omega 6 c$ & ND & $\operatorname{Tr}$ & $\operatorname{Tr}$ & ND \\
\hline iso- $\mathrm{C}_{16: 1} \mathrm{H}$ & $\mathrm{ND}$ & $1 \cdot 5$ & $1 \cdot 5$ & $2 \cdot 7$ \\
\hline $\mathrm{C}_{16: 1} \omega 7 c$ alcohol & ND & ND & ND & $\operatorname{Tr}$ \\
\hline iso- $\mathrm{C}_{16: 0}$ & $\mathrm{ND}$ & $\mathrm{ND}$ & $\operatorname{Tr}$ & $2 \cdot 1$ \\
\hline anteiso- $\mathrm{C}_{16: 0}$ & $1 \cdot 9$ & ND & ND & ND \\
\hline Summed feature $4 \dagger$ & $13 \cdot 8$ & $13 \cdot 1$ & $21 \cdot 4$ & $29 \cdot 8$ \\
\hline $\mathrm{C}_{16: 1} \omega 5 c$ & $15 \cdot 0$ & $3 \cdot 7$ & $7 \cdot 9$ & $23 \cdot 3$ \\
\hline $\mathrm{C}_{16: 0}$ & $6 \cdot 4$ & ND & $1 \cdot 7$ & $1 \cdot 1$ \\
\hline iso- $\mathrm{C}_{15: 0} 3-\mathrm{OH}$ & $\mathrm{ND}$ & $1 \cdot 6$ & $1 \cdot 6$ & $2 \cdot 7$ \\
\hline iso- $\mathrm{C}_{15: 0} 2-\mathrm{OH}$ & $\mathrm{ND}$ & $\operatorname{Tr}$ & $\operatorname{Tr}$ & $\mathrm{ND}$ \\
\hline Summed feature $5 \ddagger$ & $14 \cdot 4$ & $19 \cdot 9$ & $17 \cdot 7$ & $18 \cdot 5$ \\
\hline iso- $\mathrm{C}_{17: 0}$ & $5 \cdot 0$ & $1 \cdot 8$ & $4 \cdot 5$ & $1 \cdot 7$ \\
\hline anteiso- $\mathrm{C}_{17: 0}$ & ND & $\operatorname{Tr}$ & $2 \cdot 3$ & ND \\
\hline $\mathrm{C}_{17: 1} \omega 6 c$ & $\mathrm{ND}$ & $\operatorname{Tr}$ & ND & $1 \cdot 1$ \\
\hline iso- $\mathrm{C}_{16: 0} 3-\mathrm{OH}$ & $\mathrm{ND}$ & $\mathrm{ND}$ & ND & $1 \cdot 0$ \\
\hline $\mathrm{C}_{16: 0} 3-\mathrm{OH}$ & $\mathrm{ND}$ & $\mathrm{ND}$ & $\mathrm{ND}$ & $1 \cdot 2$ \\
\hline iso- $\mathrm{C}_{17: 0} 3-\mathrm{OH}$ & $3 \cdot 1$ & $2 \cdot 1$ & $3 \cdot 5$ & $3 \cdot 9$ \\
\hline $\mathrm{C}_{17: 0} 2-\mathrm{OH}$ & $\mathrm{ND}$ & $2 \cdot 0$ & $1 \cdot 3$ & $\mathrm{ND}$ \\
\hline
\end{tabular}

*Summed feature 2 contains one or more of the following fatty acids: iso- $\mathrm{C}_{15: 1} \mathrm{I} / \mathrm{C}_{13: 0} 3-\mathrm{OH}$.

$\dagger$ Summed feature 4 contains one or more of the following fatty acids: $\mathrm{C}_{16: 1} \omega 7 c /$ iso- $\mathrm{C}_{15: 0} 2-\mathrm{OH}$.

$\ddagger$ Summed feature 5 contains one or more of the following fatty acids: anteiso- $\mathrm{C}_{17: 1} \mathrm{~B} /$ iso $\mathrm{I}$.

\section{Acknowledgements}

This work was supported by Korea Ministry of Science and Technology under National Research Laboratory Program (M1050000011005J0000-11010).

\section{References}

Buczolits, S., Denner, E. B. M., Vybiral, D., Wieser, M., Kampfer, P. \& Busse, H. J. (2002). Classification of three airborne bacteria and proposal of Hymenobacter aerophilus sp. nov. Int J Syst Evol Microbiol 52, 445-456.

Chun, J. \& Goodfellow, M. (1995). A phylogenetic analysis of the genus Nocardia with $16 \mathrm{~S}$ rRNA gene sequences. Int J Syst Bacteriol 45, 240-245.

Collins, M. D., Hutson, R. A., Grant, I. R. \& Patterson, M. F. (2000). Phylogenetic characterization of a novel radiation-resistant bacterium from irradiated pork: description of Hymenobacter actinosclerus sp. nov. Int J Syst Evol Microbiol 50, 731-734. 
Felsenstein, J. (1981). Evolutionary trees from DNA sequences: a maximum likelihood approach. $J$ Mol Evol 17, 368-376.

Felsenstein, J. (1985). Confidence limits on phylogenies: an approach using the bootstrap. Evolution 39, 783-791.

Fitch, W. M. (1971). Toward defining the course of evolution: minimum change for a specific tree topology. Syst Zool 20, 406-416.

Hirsch, P., Ludwig, W., Hethke, C., Sittig, M., Hoffmann, B. \& Gallikowski, C. A. (1998). Hymenobacter roseosalivarius gen. nov., sp. nov. from continental Antarctic soils and sandstone: bacteria of the Cytophaga/Flavobacterium/Bacteroides line of phylogenetic descent. Syst Appl Microbiol 21, 374-383.

Jeon, Y.-S., Chung, H., Park, S., Hur, I., Lee, J.-H. \& Chun, J. (2005). jPHYDIT: a JAVA-based integrated environment for molecular phylogeny of ribosomal RNA sequences. Bioinformatics 21, 3171-3173.

Jukes, T. H. \& Cantor, C. R. (1969). Evolution of protein molecules. In Mammalian Protein Metabolism, vol. 3, pp. 21-132. Edited by H. N. Munro. New York: Academic Press.
Kovacs, N. (1956). Identification of Pseudomonas pyocyanea by the oxidase reaction. Nature 178, 703.

Marmur, J. \& Doty, P. (1962). Determination of the base composition of deoxyribonucleic acid from its thermal denaturation temperature. J Mol Biol 4, 109-118.

Saitou, N. \& Nei, M. (1987). The neighbor-joining method: a new method for reconstructing phylogenetic trees. Mol Biol Evol 4, 406-425.

Stackebrandt, E. \& Goebel, B. M. (1994). Taxonomic note: a place for DNA-DNA reassociation and 16S rRNA sequence analysis in the present species definition in bacteriology. Int J Syst Bacteriol 44, 846-849.

Staley, J. T. (1968). Prosthecomicrobium and Ancalomicrobium: new prosthecate freshwater bacteria. J Bacteriol 95, 1921-1942.

Swofford, D. L. (1998). PAUP - Phylogenetic Analysis Using Parsimony, version 4. Sunderland, MA: Sinauer Associates.

Yi, H. \& Chun, J. (2006). Thalassobius aestuarii sp. nov., isolated from tidal flat sediment. J Microbiol 44, 171-176. 